\title{
Gleichstellung der Geschlechter in der UN-Verwaltung im Bereich Friedenskonsolidierung
}

\author{
Ulrike Baumgärtner*
}

\begin{abstract}
The Security Council Resolution 1325 has changed the personal, the structure as well as the practices of UN peacekeeping. The military dominated international bureaucracy has taken efforts to increase numbers of female staff, has established gender-related institutions and has integrated gender-sensitive programs. These organizational changes can be regarded as efforts to implement the emerging norm of gender equality that shifted from the development and human rights area to the sphere of international security. This article aims at giving a systematic overview of empirical changes in the polity, politics and policy of the UN peacekeeping bureaucracy between 2000 and 2008.
\end{abstract}

Keywords: peace consolidation, gender equality, norm implementation, UN administration Friedenskonsolidierung, Gleichstellung der Geschlechter, Normimplementierung, UN-Verwaltung

\section{Einleitung}

Im letzten Jahrzehnt ist „Gender“ zu einer wichtigen Kategorie der Sicherheitspolitik geworden - oder zumindest zu einem prominenten Schlagwort. In diesem Zusammenhang hat insbesondere die Sicherheitsratsresolution (SC-Res.) ${ }^{1} 1325$ „On Women, Peace and Security“2 einige Veränderungen gebracht. Auf ihrer Grundlage hat sich vor allem die personelle Zusammensetzung, die Verwaltungsstruktur und die Praxis der Friedenskonsolidierung der Vereinten Nationen (UN) stark verändert. Mit der SC-Resolution 1325 wird angestrebt, ein ausgeglichenes Geschlechterverhältnis des UN-Personals („gender balance“) zu erreichen, spezifische Abteilungen („gender units“) für GenderFragen einzurichten und Gleichstellungsbeauftragte („gender advisor") zu bestellen. Ziel ist das „Gender-Mainstreaming“, d.h. die systematische Einbeziehung unterschiedlicher Bedürfnisse und Voraussetzungen von Männern und Frauen im Sicherheitsbereich. Der jährliche internationale Tag der UN-Peacekeeper war im vergangenen Jahr den Frauen in internationalen Einsätzen gewidmet; und auch auf der Internetseite der UN-Verwaltung ist das Thema "gender and peacekeeping “ prominent platziert. ${ }^{3}$ „Gender" ist also in aller Munde. Aber was genau heißt „Gender" im Bereich der UN-Friedenskonsolidierung?

In diesem Artikel wird beschrieben, wie sich die Norm der Geschlechtergleichstellung als Ziel von Gender-Mainstreaming im Bereich der Friedenskonsolidierung etabliert hat und an welchen beobachtbaren Veränderungen sich deren Umsetzung festmachen lässt. Dabei leistet der Artikel in zweierlei Hinsicht einen Beitrag zur Erforschung der Bedeutung von Normen in der internationalen Politik (vgl. Katzenstein 1996; Haas 1990; Finnemore/Sikkink 1998; Risse/Sikkink 1999; Schimmelfennig

* Ulrike Baumgärtner, M.A. ist wissenschaftliche Assistentin an der Universität St. Gallen und promoviert im Doktoratsprogramm „International Affairs and Political Economy“ zum Thema "Gender Equality in the UN Peacekeeping Bureaucracy“.

Eine vorherige Version des Papiers wurde bei der Jahrestagung der Schweizer Politikwissenschaft 2009 vorgestellt. Für hilfreiche Anmerkungen von Heiko Baumgärtner, Lars-Eric Cederman, Volker Franke, Heiner Hänggi, Albrecht Schnabel und David Sylvan bedanke ich mich an dieser Stelle sehr herzlich.

1 Es werden die englischen Abkürzungen verwendet, da sie im Sprachgebrauch geläufiger sind.

2 Online unter: http://www.peacewomen.org/un/sc/1325.html, 18.09.2009.

3 Online unter: http://www.un.org/Depts/dpko/dpko/info.shtml, 02.10.2009.
2003). Zum einen werden grundsätzliche Definitionen und Verständnisse von Normen, Normentwicklung und Normimplementierung aus Ansätzen der Theorien der Internationalen Beziehungen (IB) auf einen bislang wenig erforschten Politikbereich angewandt: Gleichstellung der Geschlechter in der UN-Friedenskonsolidierung. Zum anderen wird entgegen bisheriger Studien nicht die Normimplementierung auf staatlicher Ebene analysiert (vgl. True/Mintrom 2001; Finnemore 1994; Risse/Sikkink 1999). Vielmehr geht es hier um die Umsetzung der Gleichstellungsnorm innerhalb einer internationalen Verwaltung. ${ }^{4}$

Diesem Beitrag liegen folgende drei Fragen zugrunde: Was bedeutet Geschlechtergleichstellung im Kontext internationaler Sicherheitspolitik? Wer sind die Adressaten der neuen Verhaltenserwartung? Und woran erkennt man die Umsetzung von Geschlechtergleichstellung in der UN-Verwaltung zur Friedenssicherung?

\section{Gleichstellung der Geschlechter als Norm der UN-Friedenskonsolidierung}

Die Frage, wie sich die Norm der Geschlechtergleichstellung in der UN-Friedenskonsolidierung durchsetzt, bezieht sich auf die Forschungsliteratur zur Bedeutung von Normen in der internationalen Politik allgemein. Normen, definiert als kollektive Erwartungen angemessenen Verhaltens, werden generell zwei inhärente Eigenschaften zugeschrieben. Zum einen tragen sie zur Identitätsbildung von Akteuren bei („Who am I“), und zum anderen haben sie regulative Effekte auf das Verhalten der Akteure („What am I supposed to do“) (Finnemore/Sikkink 1998: 891; Katzenstein 1996: 5).

Die Gleichstellung der Geschlechter ist an sich keine neue Verhaltenserwartung in der internationalen Politik. Seit Jahrzehnten setzen sich Nichtregierungsorganisationen (NGOs) für die Stärkung von Frauen und die Gleichstellung der Ge-

4 Vgl. hierzu Arbeiten, die Normimplementierung in internationalen Organisationen untersuchen, wie z.B. Haas (1990); Locher (2007); Barnett/Coleman (2005). 
schlechter ein. ${ }^{5}$ Anfangs agierte die Frauenbewegung allerdings vornehmlich im Bereich der Entwicklungs- und Menschenrechtspolitik. Nach der Vierten Weltfrauenkonferenz in Beijing 1995 hatte sich ein internationales Netzwerk herausgebildet, bestehend aus Nichtregierungsorganisationen (NGOs), dem UN-Entwicklungsfond für Frauen (UNIFEM) und einer Reihe von Unterstützerstaaten wie z. B. Kanada und Liechtenstein, das explizit für eine Sicherheitsratsresolution als Anerkennung von Frauenrechten im Sicherheitsbereich gekämpft hat. Diese Anerkennung erfolgte im Jahr 2000 mit der SC-Res. 1325 „On Women, Peace and Security “, 6 die unter kanadischer Präsidentschaft einstimmig angenommen wurde (Shepherd 2008: 387). Hierdurch hielt die Strategie des Gender-Mainstreaming Einzug in die Debatten der internationalen Sicherheitspolitik und wurde darüber hinaus zu einer neuen handlungsanleitenden Prämisse der UN-Friedenskonsolidierung.

In der Resolution werden vier konkrete Handlungsbereiche hervorgehoben, die im Rahmen der übergeordneten Strategie der systematischen Einbeziehung unterschiedlicher Situationen und Bedürfnisse der Geschlechter und der Reflexion über das Geschlechterverhältnis in allen Bereichen der Friedenskonsolidierung unternommen werden sollen:

Erstens wird der Generalsekretär aufgefordert, gleiche Partizipationschancen von Männern und Frauen auf allen Entscheidungsebenen der Friedenskonsolidierung zu gewährleisten. Konkret gilt es, mehr Frauen als Mediatorinnen in Friedensverhandlungen einzusetzen, lokale Frauengruppen an Friedensprozessen stärker zu beteiligen, vermehrt weibliche Sonderbeauftragte des Generalsekretärs (SRSG) zu ernennen und Mitgliedstaaten aufzufordern, mehr weibliches Militär- und Polizeipersonal zu entsenden. Unter dem Stichwort „genderbalance" wird das ehrgeizige Ziel verfolgt, ein 50/50-Verhältnis von männlichem zu weiblichem Personal in Militär und Polizeiverbänden zu erreichen. ${ }^{7}$

Zweitens wird betont, dass Frauen als Teil der Zivilgesellschaft in aktuellen Konflikten in besonderem Maße gefährdet sind. Vergewaltigungen sind nicht länger Randerscheinungen, sondern werden systematisch als Strategie der Einschüchterung und Erniedrigung des Gegners eingesetzt. Hieraus leitet sich ein Bedarf ab, Frauen und Kinder in Konflikt- und Post-Konfliktsituationen insbesondere vor gender-spezifischer Gewalt vermehrt zu schützen.

Der dritte Bereich umfasst das Recht auf Strafverfolgung von Straftaten im Zusammenhang mit sexueller Gewalt und Ausbeutung. Die UN verpflichten sich, die sogenannte „Null-Toleranz Strategie“ einzuhalten. Das heißt, UN-MitarbeiterInnen dürfen sich in keiner Weise an sexueller Belästigung oder sexueller Ausbeutung gegenüber anderen UN-MitarbeiterInnen oder der lokalen Bevölkerung des Einsatzlandes beteiligen. Zudem ruft

5 Vgl. hierzu z.B. den Weltaktionsplan für Frauen (Mexiko, 1975), die „Konvention zur Eliminierung jeglicher Form von Diskriminierung von Frauen“ (1979) oder die Erklärung der Vierten Weltkonferenz für Frauen (Beijing, 1995). Für eine analytische Reflexion über die Veränderung der Zielvorstellungen in Bezug auf die Stärkung der Frauen hin zu Geschlechtergleichstellung vgl. Eyben/Napier-Moore (2009).

6 Online unter: http://www.peacewomen.org/un/sc/1325.html, 18.09.2009.

7 Siehe das Dokument "Gender Ressource Package for Peacekeeping Operations” der Planungsabteilung für Friedenseinsätze (2004), online unter: http://pbpu. unlb.org/pbps/library/GRP\%20Full\%20Version.pdf, 18.09.2009. der Sicherheitsrat alle Mitgliedsländer dazu auf, die Immunität bezüglich sexueller Gewalt und Ausbeutung aufzuheben.

Und viertens wird in der Sicherheitsratsresolution auf das inhaltliche Gender-Mainstreaming verwiesen - sprich: auf die Integration einer Geschlechterperspektive in alle Tätigkeitsfelder der Friedenskonsolidierung. Wo möglich, sollen Gleichstellungsbüros in Friedensoperationen eingerichtet, Gender-Trainings durchgeführt und bei allen Aktivitäten unterschiedliche Bedürfnisse und Voraussetzungen von Männern und Frauen berücksichtigt werden. Letzteres reicht von der Errichtung eigener sanitärer Anlagen in Einsätzen bis zur Berücksichtigung sozial-psychologischer Folgen im Rahmen von Demobilisierungsmaßnahmen (vgl. Bastick/Valasek 2008; Farr 2002). Unter Gleichstellungsnorm wird in diesem Beitrag insbesondere die personelle und inhaltliche Veränderung der Verwaltung verstanden, also die Handlungsbereiche eins und vier der Sicherheitsratsresolution. Strukturelle Veränderungen werden in der Resolution nicht explizit erwähnt.

Neben der SC-Res. 1325 wird der Zusammenhang von Geschlechtergleichstellung und nachhaltiger Friedenskonsolidierung in weiteren Erklärungen, Strategiepapieren sowie in Resolutionen der UN-Generalversammlung und des UN-Sicherheitsrats hervorgehoben. ${ }^{8}$ Ferner beziehen sich aktuelle Mandate für Friedensoperationen auf diese Grundlagendokumente und führen mitunter geschlechterspezifische Maßnahmen explizit auf. ${ }^{9}$ Die vermehrte explizite Nennung gendersensitiver Maßnahmen zeigt, dass sich die Erwartungshaltung angemessenen Verhaltens gegenüber Akteuren der UN-Friedenskonsolidierung geändert hat. „Gender“ und Sicherheit sind mittlerweile zwei Aspekte desselben Themengebiets. Somit kann man sagen, dass die Phase der Herausbildung einer neuen Norm - in diesem Fall die Verschiebung einer bestehenden Norm in einen anderen Politikbereich - abgeschlossen ist. Der sogenannte „tipping point" (Finnemore/Sikkink 1998: 901) ist überschritten.

Bevor das weitere Fortschreiten der Institutionalisierung der Gleichstellungsnorm anhand konkreter Regeln und Verfahrensweisen dargelegt wird, benenne ich im nächsten Abschnitt zunächst die wichtigsten Akteure, auf die die Erwartung, sich der Norm entsprechend „angemessen“ zu verhalten, in erster Linie gerichtet ist.

\section{Adressaten der neuen Verhaltenserwartung}

Aufgrund sich verändernder Mandate wird von den Akteuren in der UN-Friedenskonsolidierung heute mehr erwartet als nur die Überwachung eines Waffenstillstandes - wie noch zu

8 Siehe z.B. die Windhoek-Erklärung und den Namibia-Aktionsplan für die systematische Integration einer Gender-Perspektive in multidimensionalen Friedenseinsätzen (2000), das „Gender Ressource Package“ der Planungsabteilung für Friedenseinsätze (2004), den umfangreichen Bericht zu sämtlichen Aspekten von Friedenseinsätzen (2005), die Studie zu Gewalt gegen Frauen (2006), die SR-Res. 1820 „On Sexual Violence in Conflict Situations” (2008) sowie die Richtlinien zur Integration einer Gender-Perspektive in der Arbeit der UN-Polizei in Friedenseinsätzen (2008).

9 Vgl. z.B. Resolution 1509 für die UN-Mission in Liberia (2003), http:// daccessdds.un.org/doc/UNDOC/GEN/N03/525/70/PDF/N0352570. pdf?OpenElement, 13.09.2009, oder die Resolution 1590 für die UN Mission in Sudan (2005), http://daccessdds.un.org/doc/UNDOC/GEN/N05/284/08/ PDF/N0528408.pdf? OpenElement, 13.09.2009. 
Anfangszeiten der UN-Friedensoperationen. In den letzten Jahrzehnten haben sich die Friedensoperationen in komplexe, globale Unternehmungen verwandelt. Im Rahmen von Kapitel VII-Einsätzen wird z.B. von den internationalen Militäreinheiten erwartet, dass sie notfalls mit Waffengewalt ein sicheres Umfeld herstellen. Die internationale Polizei soll u.a. lokale PolizistInnen ausbilden und kriminelle Gewaltakte ahnden. Zivile Einheiten sind vor Ort, um den (Wieder-)Aufbau politischer, rechtlicher und wirtschaftlicher Strukturen zu unterstützen und somit eine nachhaltig friedliche Lage zu schaffen (Gareis/Varwick 2003; Hänggi 2005). ${ }^{10}$ Neben der Ausübung funktionaler Aufgabenbereiche wird von den Militär-, Polizeiund zivilen Einheiten zunehmend die Einhaltung eines moralisch-normativen Verhaltenskodexes erwartet. Hierunter wird nicht mehr nur der Schutz unschuldiger Zivilisten verstanden. Vielmehr stehen einzelne Menschen im Zentrum der Sicherheitspolitik. Frauen und Männer werden als zentrale Akteure angesehen, die langfristig Sicherheit gewährleisten. Die sogenannte „menschliche Sicherheit“ steht im Vordergrund und das Geschlechterverhältnis soll in all ihren Bereichen und Aktivitäten mitgedacht werden. ${ }^{11}$ Insofern wandelt sich einerseits der Aufgabenbereich, andererseits aber auch die Identität der beteiligten Akteure. Das Bild der außenstehenden, neutralen Beobachter wird sukzessive von der Vorstellung aktiver Unterstützer des Friedensprozesses abgelöst.

Konkret werden in der SC-Res. 1325 neben Mitgliedstaaten und lokalen Konfliktparteien der Generalsekretär und die UN als relevante Akteure genannt. Auf der Hauptquartiersebene, im Handlungskontext ,internationale UN“, sind die Planungsabteilung für Friedenseinsätze (DPKO) und die Abteilung für technische Unterstützung (DFS) HauptakteurInnen im Bereich der UN-Friedenskonsolidierung. Die Trennung der eher politisch ausgerichteten Abteilung zur Vorbereitung und Unterstützung von Friedenseinsätzen (DPKO) von der eher technischen Abteilung DFS, die sich um die Organisation der Logistik kümmert, erfolgte im Jahr 2007 im Rahmen der Reform der UN-Friedenskonsolidierung. ${ }^{12}$ Erstere deckt die zivile, militärische und polizeiliche Planung und Unterstützung der Friedenseinsätze ab. Bedingt durch den starken Fokus auf die Einsatzvorbereitung und -unterstützung wird die Arbeit in dieser Abteilung hauptsächlich von militärischen Führungskräften geleitet. ${ }^{13}$ Die Arbeit in der Abteilung DFS konzentriert sich auf die Bereitstellung der Einsatzlogistik. In der Unterabteilung „Verhaltenskodex und Disziplinarverfahren“ (CDU) werden zudem Gender-Trainings für alle Truppenkontingente durchgeführt. Außerdem können hier Fälle von sexueller Belästigung gegen-

10 Nach der Einteilung des ehemaligen UN-Generalsekretärs Boutros BoutrosGhali werden hier also Peacekeeping-Einsätze analysiert, wobei die jüngsten komplexen Einsätze auch Elemente des peacebuilding beinhalten, vgl.: „An Agenda for Peace. Preventing Diplomacy, Peacemaking and Peacekeeping" (1992), online unter: http://www.un.org/Docs/SG/agpeace.html, 18.09.2009.

11 Vgl. hierzu auch die Debatte zu „menschlicher Sicherheit“, die erstmals in einem Bericht der UN-Entwicklungsorganisation (1994) erwähnt wird. Für akademische Beiträge, siehe z.B. Brock (2004); Müller (2003); Paris (2001).

12 Online unter: http://www.un.org/News/Press/docs/2007/ga10602.doc.htm, 21.09.2009.

13 Siehe "Women in United Nations Peace Operations: Increasing the Leadership Opportunities" (2008), http://wiis.georgetown.edu/Publications/PeaceOpsExecSummary.pdf, 05.10.2009. über zivilen Einsatzkräften innerhalb der UN-Verwaltung (vor Ort und im Hauptquartier) gemeldet werden. ${ }^{14}$

Die Struktur des Handlungskontextes „Einsatzgebiet“ ist wesentlich weniger hierarchisch gegliedert als die internationale UN-Ebene. Lediglich der/die SRSG sowie ziviles, militärisches und polizeiliches Führungspersonal haben eine herausgehobene Position. Die ausführenden Einheiten agieren in ihren jeweiligen Funktionen gleichberechtigt nebeneinander. Der überwiegende Teil des Personals in Friedenseinsätzen, nämlich 80 bis 85 Prozent, arbeitet im militärischen oder polizeilichen Bereich. ${ }^{15}$ Allein durch das äußerlich einheitliche Auftreten wird die Gruppe der „Uniformierten“ daher häufig in der Berichterstattung und öffentlichen Wahrnehmung zusammengefasst.

\section{Umsetzung der Geschlechtergleichstellung in der UN-Friedenskonsolidierung}

In der Literatur wird die Normimplementierung meist mit dem Wandel der policy eines Staates in einem bestimmten Politikfeld gleichgesetzt (Finnemore 1994; Haas/Haas 1995; Risse/Sikkink 1999; Thakur/Weiss 2009). Die policy, hier das inhaltliche Gender-Mainstreaming, ist in diesem Artikel allerdings nur ein Aspekt der Normimplementierung. Des Weiteren finden personelle Veränderungen statt, die eine Demokratisierung der internationalen Verwaltung zum Ziel haben: Männer und Frauen sollen gleichberechtigt in allen Entscheidungsgremien mitwirken können. Somit wird auch die polity der UN-Verwaltung behandelt. Und schließlich erfolgen strukturelle Veränderungen: Gleichstellungsbüros (gender units) werden eingerichtet und Gleichstellungsbeauftragte (gender advisor) bestellt. Die Errichtung solcher gender-spezifischen Institutionen bezieht sich schließlich auf die politics der Verwaltung (siehe auch True/Mintrom 2001).

In diesem dritten Abschnitt erfolgt eine systematische empirische Aufarbeitung der Veränderungen, die seit der Verabschiedung der SC-Res. 1325 im Jahr 2000 bis zum Jahr 2008 zu beobachten waren. 2008 wurde die SC-Res. 1820 verabschiedet, eine Follow-up-Maßnahme zur SC-Res. 1325. Aus oben genannten Gründen wird Letztere für diese Analyse nicht mehr als relevant erachtet, da sie primär den Schutz vor sexueller Gewalt und nicht die Gleichstellung der Geschlechter thematisiert. Konkret geht es um Veränderungen innerhalb der beiden Abteilungen DPKO und DFS auf internationaler Hauptquartiersebene sowie in den internationalen Verwaltungen in insgesamt 14 komplexen Friedensoperationen in Einsatzgebieten, die über

14 Anklagen von Militär- und Polizeipersonal wird von den jeweiligen Entsendestaaten bearbeitet. Die CDU beobachtet allerdings soweit als möglich den Verfahrensprozess und informiert die betroffenen Personen über Ergebnisse der Verhandlungen. Online unter: http://www.un.org/Depts/dpko/CDT/about. html, 21.09.2009.

15 Vgl. hierzu die statistischen Details zu den einzelnen Einsätzen, online unter: http://www.un.org/Depts/dpko/dpko/currentops.shtml\#africa, 05.10.2009. 
eine militärische, polizeiliche und zivile Einheit verfügen und im gewählten Zeitraum aktiv sind bzw. waren. ${ }^{16}$

\subsection{Veränderte polity der UN-Verwaltung zur Friedenskonsolidierung}

Die Einführung der Gleichstellungsnorm hinsichtlich der personellen Zusammensetzung der UN-Verwaltung (gender balance) hat zusätzliche Regeln für die Personalpolitik zur Folge.

Die UN-Verwaltung auf Hauptquartiersebene ist strikt hierarchisch gegliedert: Generalsekretär - Unter-Generalsekretär (USG) - Assistenz-Generalsekretär (ASG) - Direktor 2 (D2) - Direktor 1 (D1) - Professionelle MitarbeiterIn 5 (P5) - P4 - P3 - P2 - P1 - UN Volontäre (UNV). Ab der Direktorenebene ist die Besetzung eine politische Entscheidung. Die Forderung der erhöhten Frauenbeteiligung bezieht sich nicht nur auf die Anzahl, sondern auch auf die Position der Mitarbeiterin. Im Jahr 2008 gab es im UN-Sekretariat nur auf P2-Ebene ein ausgeglichenes Geschlechterverhältnis (51,6 Prozent Frauen). Je hochrangiger die Position, desto größer der Überhang an männlichem Personal. ${ }^{17}$ Generell ist jedoch auf allen Ebenen eine Tendenz zu erkennen, die darauf hindeutet, dass der Frauenanteil langfristig steigen wird (vgl. Tabelle 1).

Neben dem Appell an die Mitgliedsländer, speziell für hochrangige Posten Frauen vorzuschlagen, hat sich jüngst die informelle Regel durchgesetzt, dass auf jede sogenannte „short list“, auf deren Grundlage Sonderbeauftragte des Generalsekretärs für Einsätze ernannt werden, auch eine Frau mit aufgenommen wird. ${ }^{18}$ Insgesamt bekleideten bislang acht Frauen Führungspositionen in UN-Einsätzen (vgl. Tabelle 1). Bei den übrigen Postenbesetzungen gilt: Bei gleicher Qualifikation werden Frauen bevorzugt. Allerdings werden keine proaktiven Maßnahmen ergriffen, um den Frauenanteil zu erhöhen. ${ }^{19}$ Eine Quoteneinführung wird in diesem Zusammenhang kontrovers diskutiert. BefürworterInnen betonen, dass die UN sehr bürokratisch organisiert sei und Veränderungen nur durch Druck umgesetzt würden. GegnerInnen führen an, dass Frauen innerhalb des Teams ein geringeres Ansehen genießen würden, weil sie nicht allein wegen ihrer fachlichen Kompetenz, sondern aufgrund einer Quotenregelung angestellt wären. ${ }^{20}$

16 Im Einzelnen handelt es sich um folgende UN-Missionen: UNFICYP (Zypern), MINURSO (Westliche Sahara), UNOMIG (Georgien), UNMBIH (Bosnien-Herzegowina), UNMIK (Kosovo), UNTAET (Ost-Timor), UNAMSIL (Sierra Leone), MONUC (Demokratische Republik Kongo), UNMISET (Ost-Timor), UNMIL (Liberia), UNOCI (Côte d'Ivoire), ONUB (Burundi), MINUSTAH (Haiti), UNMIS (Sudan). Hybride Einsätze, die von mindestens zwei internationalen Organisationen ausgeführt werden, wie z.B. die UN/EU-Mission MINUCRAT in der Zentralafrikanischen Republik und Tschad oder die UN/AU-Mission UNAMID im Sudan, werden nicht berücksichtigt.

17 Online unter: http://www.un.org/womenwatch/osagi/fpgenderbalancestats. htm, 21.09.2009.

18 Siehe "Women in United Nations Peace Operations: Increasing the Leadership Opportunities" (2008), S.36, online unter: http://wiis.georgetown.edu/ Publications/PeaceOpsExecSummary.pdf, 05.10.2009.

19 Siehe die Forderungen im "Gender Resource Package for Peacekeeping Operations" der DPKO von 2004, online unter: http://www.un.org/Depts/dpko/ lessons/, 05.10.2009.

20 Die Einschätzungen basieren auf 22 qualitativen Experteninterviews mit MitarbeiterInnen aller funktionalen Einheiten in der UN-Verwaltung auf Hauptquartiers- und lokaler Einsatzebene, die ich im April 2009 durchgeführt habe.
Auf der Ebene der Friedenseinsätze kommt für die Normumsetzung erschwerend hinzu, dass Angehörige der Militär- und Polizeieinheiten von den Mitgliedstaaten entsandt werden. Nahezu ein Drittel des gesamten militärischen und polizeilichen Personals kommt aus Pakistan, Bangladesch und Indien. Leider liegen keine statistischen Daten vor, wie hoch der Anteil der Frauen an den Kontingenten dieser Länder ist. In Liberia hat allerdings eine rein weibliche Polizeieinheit aus Indien für großes Aufsehen gesorgt. ${ }^{21}$ Die Einschätzungen, ob rein weibliche Einheiten Vorbildcharakter haben, gehen auseinander. Positiv wird von BefürworterInnen hervorgehoben, dass eine so massive Präsenz anders wahrgenommen und die Frauen schneller respektiert würden als wenige Frauen in einer ansonsten männlich dominierten Einheit. Manche Frauen haben sich auch nur für den Einsatz gemeldet, weil sie wussten, dass die Truppe rein weiblich sein wird. Ferner würde die UN auf diese Weise ihrer Vorbildfunktion gerecht, lokale Frauen zu ermutigen, sich ebenfalls für öffentliche Ämter zu bewerben oder der Polizei beizutreten. Skeptische Stimmen meinen, dass es eher ein glücklicher Zufall gewesen sei, dass die Sicherheitslage nicht eskalierte; eine rein weibliche Truppe könne der Sicherheitssituation und den kulturellen Bedingungen nicht gerecht werden.

Insgesamt fällt auf, dass auf allen Ebenen der UN-Verwaltung der Frauenanteil tendenziell ansteigt. Die starken Schwankungen auf D2-Ebene sind bemerkenswert. Auf den unteren Ebenen des Hauptquartierpersonals ist eine "gender-balance“ erreicht. Auch der Frauenanteil des uniformierten Personals in Einsätzen ist ganz überwiegend steigend; in MINURSO um 1,4 Prozent. Lediglich die Missionen in Georgien und Kosovo haben sinkende oder gleichbleibende Frauenanteile.

\subsection{Veränderte politics der UN-Verwaltung zur Friedenskonsolidierung}

Der zweite Bereich der Normimplementierung bezieht sich auf die institutionellen Veränderungen (vgl. True/Mintrom 2001). Nach Verabschiedung der SC-Res. 1325 erfolgte in einem sehr beeindruckenden Tempo die Einrichtung spezifischer Gleichstellungsbüros und die Bestellung von Gleichstellungsbeauftragten. In den Gleichstellungsbüros arbeiten mehrere Gleichstellungsbeauftragte oder sogenannten „Gender-BeraterInnen“. Sie setzen sich bereits in der Planungsphase von Einsätzen für die Integration einer Geschlechterperspektive ein. Konkrete Maßnahmen reichen von der Errichtung separater sanitärer Anlagen über die Erhöhung des Frauenanteils in den Abteilungen bis hin zur Kontaktaufnahme und Einbeziehung lokaler Frauenorganisationen. Die dritte Variante geschlechterbezogener struktureller Veränderung ist die Ernennung sogenannter „gender focal points". Im Unterschied zu den hauptamtlichen Gleichstellungsbeauftragten arbeiten die focal points in thematisch unterschiedlichen Bereichen, wie etwa Entwaffnung, Sicherheitssektorreform etc. Sie verpflichten sich neben dieser Tätigkeit freiwillig, Gender-Mainstreaming voranzutreiben. In der Praxis sind sie häufig erste AnsprechpartnerInnen bei unfairer

21 Online unter: http://www.un.org/apps/news/printnewsAr.asp?nid=25557, 21.09.2009. 
Tabelle 1: „Gender Balance“ in der UN-Friedenskonsolidierung ${ }^{1}$

\begin{tabular}{|c|c|c|c|c|}
\hline \multicolumn{5}{|c|}{ Personaldaten für die Hauptquartiersebene $^{2}$} \\
\hline & 1998 & 2001 & 2006 & 2008 \\
\hline USG & $11,1 \%$ & $12,1 \%$ & - & $17,5 \%$ \\
\hline ASG & $13 \%$ & $10,8 \%$ & - & $23,5 \%$ \\
\hline D2 & $18,8 \%$ & $19,4 \%$ & $30,4 \%$ & $23,8 \%$ \\
\hline D1 & $23,7 \%$ & $29,6 \%$ & $25,3 \%$ & $28,7 \%$ \\
\hline P5 & $29,3 \%$ & $29,9 \%$ & - & $31,6 \%$ \\
\hline P4 & $32,3 \%$ & $31,5 \%$ & $35,9 \%$ & $35,9 \%$ \\
\hline P3 & $39,4 \%$ & $37,5 \%$ & - & $40,5 \%$ \\
\hline P2 & $45,4 \%$ & $47 \%$ & - & $51,6 \%$ \\
\hline P1 & $64,3 \%$ & $67,9 \%$ & - & - \\
\hline \multicolumn{5}{|c|}{ Personaldaten für Führungspersonal im Einsatz ${ }^{3}$} \\
\hline SRSG & $\begin{array}{l}\text { 1998-99: } \\
\text { Gro Harlem Brundtland } \\
\text { (UNFICYP, Zypern) }\end{array}$ & $\begin{array}{l}\text { 1995-2001: } \\
\text { Elizabeth Rehn (UNM- } \\
\text { BIH, Bosnien-Herzego- } \\
\text { wina) }\end{array}$ & $\begin{array}{l}\text { 2002-2006: } \\
\text { Heidi Tagliavini (UN- } \\
\text { OMIG, Georgien) } \\
\text { 2004-2006: } \\
\text { Carolyn McAskie } \\
\text { (ONUB, Burundi) }\end{array}$ & $\begin{array}{l}\text { seit 2007: } \\
\text { Ellen Margrethe Løj } \\
\text { (UNMIL, Liberia) }\end{array}$ \\
\hline DSRSG & \multicolumn{3}{|c|}{ keine weibl. DSRSG } & $\begin{array}{l}\text { seit 2007: } \\
\text { Henrietta Joy Abena Nyarko } \\
\text { Mensa-Bonsu (UNMIL, } \\
\text { Liberia) } \\
\text { seit 2008: } \\
\text { Leila Zerrougui (MONUC, DR } \\
\text { Kongo) } \\
\text { seit 2008: } \\
\text { Ameerah Haq (UNMIS, } \\
\text { Sudan) } \\
\end{array}$ \\
\hline $\begin{array}{l}\text { Militärische } \\
\text { Einsatzleitung }\end{array}$ & \multirow{2}{*}{\multicolumn{4}{|c|}{ kein weibliches Personal }} \\
\hline $\begin{array}{l}\text { Polizeiliche } \\
\text { Einsatzleitung }\end{array}$ & & & & \\
\hline \multicolumn{5}{|c|}{ Daten für uniformiertes Einsatzpersonal } \\
\hline & & 1993 & 2006 & 2008 \\
\hline \multirow{10}{*}{ Militäreinheiten } & UNFICYP, Zypern & $0,7 \%$ & $6,3 \%$ & $7,7 \%$ \\
\hline & MINURSO, Westsahara & $10,2 \%$ & $3,7 \%$ & $5,1 \%$ \\
\hline & UNOMIG, Georgien & $0 \%$ & $4,1 \%$ & $3,7 \%$ \\
\hline & UNMIK, Kosovo & 1999 errichtet & $0 \%$ & $0 \%$ \\
\hline & MONUC (DR Kongo) & 1999 errichtet & $2 \%$ & $1,5 \%$ \\
\hline & \begin{tabular}{|l|} 
UNMIL, Liberia \\
\end{tabular} & 2003 errichtet & $2,3 \%$ & $3,3 \%$ \\
\hline & UNOCI, Elfenbeinküste & 2004 errichtet & $0,6 \%$ & $1,3 \%$ \\
\hline & ONUB, Burundi & 2004 errichtet & $4,7 \%$ & 2006 aufgelöst \\
\hline & MINUSTAH, Haiti & 2004 errichtet & $1,4 \%$ & $1,8 \%$ \\
\hline & UNMIS, Sudan & 2005 errichtet & $1 \%$ & $1,4 \%$ \\
\hline \multirow{3}{*}{ Polizeieinheiten } & 16,7\% UNFICYP, Zypern & \multirow{3}{*}{\multicolumn{3}{|c|}{ keine statistischen Daten für den Zeitraum vorhanden }} \\
\hline & 0\% MINURSO, Westl. Sahara & & & \\
\hline & 0\% UNOMIG, Georgien & & & \\
\hline
\end{tabular}

\section{Quellen:}

Peacewomen (NGO): http://www.peacewomen.org/un/pkwatch/facts.html, 05.10.2009.

UN-Planungsabteilung für Friedenseinsätze: http://www.un.org/Depts/dpko/dpko/contributors/gend.html, 05.10.2009.

Office of the Special Advisor on Gender Issues and Advancement of Women: http://www.un.org/womenwatch/osagi/pdf/status\%20of\%20 women\%20in\%20UN\%20 Secretariat.pdf, 05.10.2009.

Division of the Advancement of Women (1995): The role of women in United Nations peace-keeping, Women2000, No.1.

1 Die Prozentzahl gibt den Anteil weiblichen Personals in den Gremien an. Leider existieren keine fortlaufenden Statistiken für alle Bereiche.

2 Die statistischen Daten der UN-Verwaltung auf Hauptquartiersebene beziehen sich auf das gesamte UN-Sekretariat, nicht nur auf die Abteilungen für Friedenskonsolidierung.

3 Es werden nur Einsätze gemäß oben genannter Kriterien berücksichtigt. 
Behandlung im Rahmen von Rekrutierungen und in Fällen von sexueller Belästigung am Arbeitsplatz. ${ }^{22}$

Auf der internationalen UN-Ebene existieren seit 2003 eine P5und eine P3-Stelle für Gleichstellungsbeauftragte. UN-Polizeiund Militär-Division in New York verfügen ebenfalls über jeweils eine „Gender-Beraterin“, wobei die Gender-Beraterin der UN-Polizei nicht aus dem allgemeinen Budget, sondern separat von der Schweiz finanziert und entsandt wird. Darüber hinaus wurden in den meisten Abteilungen von DPKO focal points ernannt. Das Netzwerk „Frauen, Sicherheit und Frieden“ ist quasi die Fortführung der Lobbygruppe für die SR-Res. 1325. Das Netzwerk besteht aus Vertreterinnen unterschiedlicher UNAbteilungen, NGOs und staatlichen Repräsentanten und trifft

22 Siehe die Ausführungen des „Gender Resource Package“, S. 6f. und S. 33, online unter: http://www.un.org/Depts/dpko/lessons/, 05.10.2009. sich regelmäßig zum Erfahrungsaustausch und zur Planung neuer Initiativen und Kampagnen.

Auf Einsatzebene existieren gemäß Recherchen der NGO Peacewomen in den UN-Einsätzen in Zypern, der Westsahara und Georgien jeweils gender focal points. Im Kosovo, der Elfenbeinküste und im Sudan sind Gleichstellungsbeauftragte eingestellt und in den Missionen in der Demokratischen Republik Kongo, Liberia und Haiti Gleichstellungsbüros mit mehreren Personalstellen eingerichtet. Beeindruckend ist, dass alle diese strukturellen Veränderungen innerhalb der UN-Verwaltung und in den Einsätzen im Jahr 2000 erfolgten, also noch im Jahr der Verabschiedung der Resolution $1325 .{ }^{23}$ Es wird allerdings bemängelt, dass zu wenig Ressourcen aufgebracht würden, die Stellen also

23 Online unter: http://www.peacewomen.org/un/pkwatch/facts.html, 05.10.2009.

Abb. 1: Strukturelle Veränderungen für mehr Gleichstellung

Generalsekretär

Sekretariat

- Planungsabteilung für Friedenseinsätze (DPKO)

- Gleichstellungsbüro im Bereich Best Practice

- Militärische Abteilung (mit Gleichstellungsbeauftragter $/ m$ )

- Polizeiabteilung (mit Gleichstellungsbeauftragte/m)

- Etc.

- Abteilung für technische Unterstützung (DFS)

- Abteilung zur logistischen Einsatzunterstützung

- Abteilung für Verhaltenskodexe und Disziplinarverfahren

- Etc.

HANDLUNGSKONTEXT: INTERNATIONALE UN

UN-Führungspersonal

- SonderbeauftragteR des Generalsekretärs (SRSG)

- StellvertretendeR SonderbeauftragteR (DSRSG)

- Militärische Einsatzleitung

- Polizeiliche Einsatzleitung

Netzwerk für

Frauen,

Sicherheit und

Frieden

\section{- Militäreinheit}

Kontingente aus Mitgliedsländern

lokale

MitarbeiterInnen

\section{- Polizeieinheit}

- $\quad$ Kontingente aus Mitgliedsländern

- lokale

MitarbeiterInnen

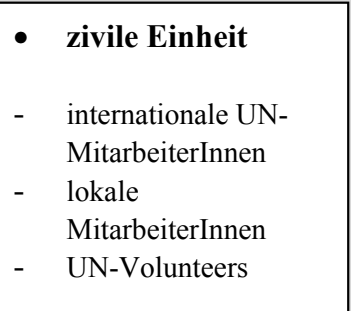

Gleichstellungs-

büro/

Gleichstellungs-

beauftragteR

HANDLUNGSKONTEXT: EINSATZGEBIET 
zu niedrig dotiert seien. Außerdem seien die Kompetenzen des Personals zu gering für den umfangreichen Aufgabenkatalog, den es zu bewältigen gelte (Rehn/Sirleaf 2002).

Zur besseren Einordnung sind in der folgenden Abbildung die neuen genderbezogenen Büros und Anlaufstellen in die Übersicht der UN-Verwaltung zur Friedenskonsolidierung eingetragen.

\subsection{Veränderte policy der UN-Verwaltung zur Friedenskonsolidierung}

Schließlich drückt sich die Umsetzung der Gleichstellungsnorm in sich verändernden Debatten, Richtlinien und Aktivitäten aus (vgl. Finnemore 1994; Locher 2007). Auf internationaler UN-Ebene wird die inhaltliche Veränderung zu vermehrter Geschlechtergleichstellung z.B. in der jährlichen Sicherheitsdebatte zu Frauen, Frieden und Sicherheit und dem jährlichen Bericht des Generalsekretärs zum Thema deutlich. Es gibt eine Reihe von Policy-Berichten, welche die Integration verschiedener Aspekte von Gender-Mainstreaming thematisiert. ${ }^{24}$ Aber auch Dokumente und Debatten, die nicht speziell auf die Umsetzung von Geschlechtergleichstellung ausgerichtet sind, integrieren vermehrt eine Gender-Perspektive. So führen die UN seit 2006 eine Gender-Statistik für das uniformierte Einsatzpersonal. Außerdem enthalten die allgemeinen Richtlinien zu UN-Friedenseinsätzen nunmehr auch Abschnitte zu „gender and peacekeeping “. 25

Hinsichtlich des Handlungskontextes des Einsatzgebiets sind alle MitarbeiterInnen der UN-Friedensoperationen verpflichtet, ein spezielles Gender-Training zu durchlaufen. Wird ein solches Training von den Mitgliedstaaten nicht angeboten, übernimmt das DFS diese Zusatzausbildung. Zudem werden bestimmte Schulungen vor Ort durchgeführt, die auf die jeweilige Situation und die kulturellen Besonderheiten im Umgang zwischen den Geschlechtern eingehen. Denn in komplexen UN-Friedensoperationen beinhaltet der Aufgabenkatalog der Militär- und Polizeieinheiten mehr als die traditionelle Überwachung von Waffenstillständen oder die robuste Gewaltanwendung zur Herstellung eines sicheren Umfelds. Uniformiertes UN-Personal sucht vermehrt Kontakt zur lokalen Bevölkerung, bildet lokale Sicherheitskräfte aus und unterstützt mitunter Entwicklungsprojekte in logistischer Hinsicht. Um den Herausforderungen angemessen begegnen zu können, gilt die Integration von Frauen in UN-Militär und -Polizei häufig als Notwendigkeit. Sicherheitskontrollen an Frauen oder Durchsuchungen von Frauenhäusern können nur von weiblichem Personal ausgeführt werden. Ebenso ist es nach Ansicht der meisten Beteiligten weiblichen Opfern von sexuellen Gewalttaten nicht zumutbar, von männlichem Personal vernommen zu werden. Die Beteiligung von Frauen und die Berücksichtigung geschlechtsspezifischer Situationen vor Ort erhöhen die

24 Wie etwa das „Gender Ressource Package for Peacekeeping Operations”, online unter: http://www.un.org/Depts/dpko/lessons/, 05.10.2009.

25 Gender Statistik, online unter: http://www.un.org/Depts/dpko/dpko/contributors/gender.shtml, 05.10.2009; Richtlinien zu UN-Einsätzen online unter: http://pbpu.unlb.org/pbps/Library/Capstone_Doctrine_ENG.pdf, 05.10.2009.
Effektivität komplexer Friedenseinsätze, so die Meinung vieler UN-Mitarbeiter. ${ }^{26}$

\section{Abschließende Bemerkungen}

Ausgangspunkt dieses Beitrags ist die Beobachtung, dass „Gender" im Bereich der UN-Friedenskonsolidierung zu einem regelrechten Leitmotiv geworden ist. Mit der Resolution 1325 thematisierte erstmals eine Resolution des UN-Sicherheitsrats die Geschlechterperspektive in diesem Politikbereich. Ziel des Beitrags war es, die formalen Veränderungen als Folge der Normimplementierung systematisch darzustellen. Als Ergebnis ist festzuhalten, dass seit 2000 deutliche Veränderungen hin zu einer vermehrten Gleichstellung der Geschlechter in der personellen Zusammensetzung, der Organisationsstruktur und der inhaltlichen Programmatik der UN-Verwaltung zur Friedenskonsolidierung zu beobachten sind. Dies ist insbesondere bemerkenswert, da der Bereich der Friedenskonsolidierung traditionell stark militärisch ausgerichtet ist und das Führungspersonal noch mehrheitlich aus den Reihen des Militärs rekrutiert wird. Kaum ein anderes Tätigkeitsfeld wird so stark mit Männlichkeit assoziiert wie der Militäreinsatz und die Entsendung von internationalen Militär- und Polizeieinheiten in Krisengebiete. Insofern widerspricht dieses Ergebnis der intuitiven Vermutung, die Umsetzung der Norm der Geschlechtergleichstellung würde im Bereich der UN-Friedenskonsolidierung aufgrund einer mangelnden Akzeptanz nicht oder nur schleppend erfolgen. Die hier vorgelegte Analyse kann nur einen ersten Ausgangspunkt für die weitere Beschäftigung mit dem Thema darstellen. So wäre nicht zuletzt auch nach der subjektiven Wahrnehmung der beteiligten Akteure hinsichtlich der Durchsetzung der Gleichstellungsnorm zu fragen. Auch die Frage nach den kausalen Mechanismen, die schließlich zur Umsetzung der Geschlechtergleichsetzung führen, erscheint in diesem Zusammenhang relevant. All dies wäre Gegenstand weiterer Untersuchungen.

\section{Literatur}

Barnett, Michael/Coleman, Liv 2005: Designing Police: Interpol and the Study of Change in International Organizations, in: International Studies Quarterly 49: 593-619.

Bastick, Megan /Valasek, Kristin 2008: Gender \& Security Reform Toolkit, online unter: http://www.dcaf.ch/gender-security-sector-reform/gssr-toolkit.cfm, 05.10.2009.

Brock, Lothar 2004: Der erweiterte Sicherheitsbegriff: Keine Zauberformel für die Begründung ziviler Konfliktbearbeitung, in: Die Friedenswarte 79: 3-4, 323-343.

Eyben, Rosalind/Napier-Moore, Rebecca 2009: Choosing Words with Care? Shifting meanings of Women's Empowerment in International Development, in: Third World Quarterly 30: 2, 285-300.

26 Diese Einschätzung basiert auf den geführten Experteninterviews (siehe oben). 
Farr, Vanessa 2002: Gendering Demilitarization as Peacebuilding Tool, online unter: http://unddr.org/docs/Gendering\%2 0Demilitarization\%20as\%20a\%20Peacebuilding\%20Tool.pdf, 05.10.2009.

Finnemore, Martha 1994: International Organizations as Teachers of Norms: The United Nations Educational, Scientific, and Cultural Organization and Science Policy in: International Organization 47: 4, 565-597.

Finnemore, Martha/Sikkink, Kathryn 1998: International Norm Dynamics and Political Change, in: International Organization 52: 4, 887-917.

Gareis, Sven Bernhard/Varwick, Johannes 2003: Die Vereinten Nationen. Aufgaben, Instrumente und Reformen, Opladen: Leske+Budrich.

Haas, Ernst B. 1990: When Power is Knowledge. Three Models of Change in International Organizations, Berkeley: University of California Press.

Haas, Peter M./Haas, Ernst B. 1995: Learning to Learn: Improving International Governance, in: Global Governance 1: 255-285.

Katzenstein, Peter J. 1996: The Culture of National Security: Norms and Identity in World Politics New York: Columbia University Press.

Locher, Birgit 2007: Trafficking in Women in the European Union. Norms, Advocacy-Networks and Policy-Change, Wiesbaden: VS Verlag für Sozialwissenschaften.

Müller, Harald 2003: Begriff, Theorien und Praxis des Friedens, in: Hellman, Gunter/Wolf, Klaus Dieter /Zürn, Michael (Hrsg.):
Die neuen Internationalen Beziehungen. Forschungsstand und Perspektiven in Deutschland, Baden-Baden, 209-250.

Paris, Roland 2001: Human Security. Paradigm Shift or Hot Air?, in: International Security 26: 2, 87-102.

Rehn, Elisabeth/Sirleaf, Ellen Johnson 2002: Women, War and Peace: The Independent Experts' Assessment on the Impact of Armed Conflict on Women and Women's Role in Peacebuilding, online unter: http://www.unifem.org/materials/item_detail.php?ProductID=17

Risse, Thomas/Sikkink, Kathryn 1999: The Socialization of International Human Rights Norms into Domestic Practices: Introduction, in: Risse, Thomas/Ropp, Stephen C./Sikkink, Kathryn (Hrsg.): The Power of Human Rights. International Norms and Domestic Change, Cambridge, Cambridge University Press.

Schimmelfennig, Frank 2003: The EU, NATO and the Integration of Europe. Rules and Rhetoric, Cambridge: Cambridge University Press.

Shepherd, Laura J 2008: Power and Authority in the Production of the United Nations Security Council Resolution 1325, in: International Studies Quarterly 52: 383-404.

Thakur, Ramesh /Weiss, Thomas G. 2009: United Nations "Policy": An Argument with Three Illustrations in: International Studies Perspectives 10: 10, 18-35.

True, Jacqui/Mintrom, Michael 2001: Transnational Networks and Policy Diffusion: The Case of Gender Mainstreaming, in: International Studies Quarterly 45: 1, 27-57.

\title{
Sicherheit und Menschenrechte
}

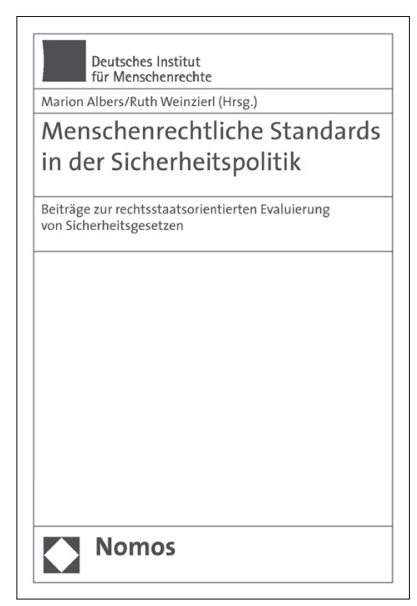

\author{
Menschenrechtliche Standards in der Sicherheitspolitik \\ Beiträge zur rechtsstaatsorientierten Evaluierung von Sicherheitsgesetzen \\ Herausgegeben von Marion Albers und Ruth Weinzierl \\ 2010, 198 S., brosch., 29,- $€$, ISBN 978-3-8329-4337-O
}

Evaluationen sicherheitsbehördlicher Kompetenzen haben sich mittlerweile zu einem zentralen Baustein des neuen Sicherheitsrechts entwickelt. Dieser Band bietet erstmals einen Überblick über Hintergründe, Grundlagen, methodische Ansätze, bisherige Erfahrungen und spezifische Problemstellungen bei der Evaluierung von Sicherheitsgesetzen.

Bitte bestellen Sie im Buchhandel oder unter $\downarrow$ www.nomos-shop.de 